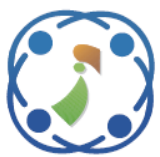

\title{
Cluster Based K-Medoids Approach to Discriminate Travelling Time in Wireless Sensor Network
}

\author{
Raja Murali Prasad ${ }^{1 *}$ \\ Gandluru Arthur Edwin Satish Kumar ${ }^{1}$ \\ ${ }^{1}$ Department of Electronics and Communication Engineering, Vardhaman College of Engineering, \\ Hyderabad, Telangana, India \\ * Corresponding author’s Email: rajamurali.prasad12@gmail.com
}

\begin{abstract}
As cluster heads chosen by nodes, gathers the data from their individual groups and exchanges the collected data to the mobile sink as proposed in the heuristic tour planning technique. In this research, the mobile sink starts the collection of data irregularly from the initial site, which is stored in cluster heads in an individual hop extend and at last returns to the initial site. In existing approaches, scheduling time for data delivery is less. To increase the data delivery, there is a need in extending the concept to higher level routing process. To solve this issue, this paper proposed a collection oriented distribution scheme with k-medoids (CODS-KM). The method can be exhausted for distribute the collection points in network in a particular manner. In this method, k-medoids algorithm for clustering and cluster head process is used. To collect the data purpose, it can be used $\mathrm{CH}$ (Cluster Head), CP (Collection Point) and RP (Rendezvous Point). The proposed system helps in mitigation of travelling time when compared to existing approaches. To find the effectiveness of proposed algorithm, NS2 (Network simulator-2) software is used for simulation verification.
\end{abstract}

Keywords: Wireless sensor networks, Energy efficient routing, Cluster-based approach, Mobile sink, K-medoids, Tour planning approach, Collection oriented mechanism.

\section{Introduction}

Wireless Sensor Networks have been introduced in many domains including health care systems, natural conditions monitoring, military applications, and others as Internet of Things [1-3, 5]. The most key issue for wireless sensor networks is energy efficiency. However, the supply of power is limited and hard to replace for sensor nodes. And also, nodes close to the base station expend more energy in contrasted and different nodes since the nodes transfer the information received by sensor nodes stay away from the sink. Henceforth, the information received by different sensors can't be exchanged to the sink once these sensors close to the sink fail. The complete setup ends up early at that particular point, even though a large part of the nodes can even now have an expected measure of energy. In addition to that, restricting the energy utilization of sensor nodes is the key failures for
WSNs to expand the system lifetime. In [4], WSN module of the detecting nodes utilized as a part of this work was provided by TI with cutting edge qualities of the WSN, for example, adaptability, adaptation to non-critical failure, high detecting constancy and quick sending utilizing ZigBee convention.

A sensor organize is categorized as a making of a substantial number of less effort, low power multi utilitarian sensor nodes that are optionally conveyed either inside the system or near it. These nodes which are small in measure involve detecting, data preparing and communication parts. The direction of these small nodes require not be superior; this provides irregular condition as well as implies that conventions of such sensor systems and its related outputs must have self sorting out ability in out of reach zones. However, an exceptionally best among the most fundamental limitations on sensor nodes are the low power utilization necessities, nodes are 
constrained in power supply and data exchange limit. These limitations have pointed diverse problems to the design and administration of systems joined with a specific plan of considerable number of nodes. These problems require power saving. Consequently the exploration on these territories has been marked around system level power mindfulness, for example, dynamic voltage scaling, radio correspondence system, low cycle issues, system portioning, and energy aware MAC (Medium access controller) protocols, the problems identified with physical and connect layers are huge and normal for all types of sensor applications. The solid exchanging of data from the sensor nodes to the sink and the principle point is to find paths for power effective path setup at the system layer with the objective that the lifetime of the system is helped.

In this exploration, it is attempted to find designing issues and highlight their recommendations since the execution of a coordinating protocol is more related to the building model.

\subsection{Process of network}

There are three principle fragments in a sensor arrangement. These are the sensor nodes, sink and checked events. Alongside the not a lot of setups that utilize portable sensors, most by far of the system configuration expects that sensor nodes are stationary. Then even supporting the mobility of sink or cluster heads is some of the time considerable conditions.

\subsection{Placement of node}

Other thought is the topological plan of the nodes which is application based and impacts the execution of the routing protocol. The design is either deterministic or self managing. In deterministic conditions, the sensors are physically put and data is coordinated through already determined techniques. However in self dealing with system the sensor nodes are arranged arbitrarily that makes a base in a specially appointed way.

\subsection{Manipulation of energy levels}

In the development of a system, an algorithm of aligning the paths is significantly affected from energy considerations. As the remote radio transmission energy is corresponding to the distance coincide or substantially more advanced request within the sight of obstacles, multi hop routing can consume low energy when compared to coordinate analogy. Be that as it may, multi hop routing presents moderate controlling access as well as huge upward topology rule. Coordinate directing can act well advice when every one of the nodes approaches the sink. Hence sensors are not scattered conditionally finished a zone of intrigue and multi hop directing winds up plainly unavoidable.

\subsection{Packet flow practices}

Based on the utilization of the sensor arrange, an information conveyance model to the sink will be persistent, event driven, inquiry driven and half breed. In constant conveyance show, every sensor sends information intermittently. In event driven and question driven models, when an occasion happens or an inquiry is produced by the sink, the transmission of information is activated in object controlled models and event controlled models. Some system applies a crossover arrange utilizing a mix of nonstop, event driven and question driven information delivery. The routing protocol is very impacted by information delivery model, particularly concerning the minimization of energy utilization and route security [6].

In this paper, the mobile sink will travel through the system with obstacles to discover an impediment keeping away from most brief route. In the meantime, the mobile sink must consider the vitality utilization adjust among nodes while moving over the detecting field. To dispatch the portable sink proficiently, it is used the cluster based technique that is exhibited in [7] and [8]. As indicated by the cluster based technique, all sensor nodes in the detecting field are partitioned into two classes: cluster heads and cluster individuals. Cluster heads gather information from relating group individuals which gather condition data, and after that pass information to the mobile sink. It can be expected that WSNs can endure some degree of delay with the goal that the mobile sink gathers all detecting information from cluster heads. The portable sink starts its periodical development from beginning site lastly returns. Amid its development, the portable sink gathers the detecting information from cluster heads. When it's moving way is arranged, the mobile sink can move close to the group heads and devour less energy. Henceforth, the system lifetime can be drawn out fundamentally.

In this paper, the system lifetime is characterized as the time interim from sensor nodes begin working until the passing of every static sensor. However, in physical situations, the detecting field may contain different obstacles which make the planning for the portable sink more mind boggling. Here, the portable sink can move to any site aside from the site of obstacles. Subsequently, an exploration challenge is the manner by which to effectively 
dispatch the mobile sink to discover an obstacle maintaining a strategic distance from most brief route within the sight of obstructions.

To illuminate the planning for the mobile sink, it found that the way to make the dispatch issue less complex in WSNs with obstacles. Given the multifaceted nature of the problem, it is presented that a grid based method by which the detecting locale is separated into a similar size lattice cells. Grid cells are considered to be the basic unit and their size is firmly identified with correspondence span of static sensors. As the two-dimensional plane is separated into a similar size matrix cells, obstructions will contain some lattice cells. Edges of obstacles cross grid cells and deterrents may involve some portion of some lattice cells. Once obstacles occupy part of one grid cell, it can be expected that the grid cell is viewed as obstructions. Subsequently, it is acquired that the regularization state of obstacles with the goal that the planning for the mobile sink ends up noticeably simpler. It is able to build a spanning diagram regarding the regularization state of obstacles. With the search space of the portable sink from all grid cells to the spreading over chart acquiring grid cells, the scheduling for the mobile sink will turn out to be more efficient. Consequently, at last it is found that an obstacle-avoiding shortest route for the mobile sink.

In this paper, cluster based travelling algorithm proposed it have specified in proposed system described in area three. In proposed work, accumulation of numerous information and put away in arrange point. Here proficiency of system can be spoken to. The k-medoids algorithm is a segregating around medoids which is slightly modified from the k-means algorithm. In k-medoids, data points are chosen to be the medoids. The object of a cluster, whose average dissimilarity to all the objects in the cluster is minimal and it can be defined as medoids. Our work is to minimize the travelling time in WSN using proposed framework named as CODS-KM scheme.

The organization of paper is as follows. Section 2 presents research study. The proposed framework is presented in section 3. Section 4 describes the experimental study of proposed paper. And finally the paper is concluded in section 5 .

\section{Research study}

The advantage of utilizing the mobility of nodes in the demonstration of recent work has been well recognized. Ease of traffic burden and enhance energy efficiency by using the mobility of nodes in
WSNs is possible. Henceforth; the system lifetime is prolonged significantly. Many papers have proposed various different approaches.

The authors present a VGDRA (virtual gridbased dynamic routes adjustment scheme) scheme to minimize the communication cost for the mobile sink in [9]. The cell-header nodes are chosen by the nodes near the center and the sensor field is isolated into a virtual grid containing the same size cells. And also, the construction of a virtual backbone structure is done which consists of the cell header nodes. By communicating with the border cell header nodes, the mobile sink collects the detecting information and moves across the sensor field. The routes remaking process incorporates just a subset of cell-header nodes to decrease the general correspondence cost. In [9], the drawbacks are same size of cells used for grid and it doesn't verify background process in network. In [10], the authors proposed a blended number programming system for base station to relieve the problematic energy scattering. To invert the problematic energy scattering patterns, the base station portability is acquainted with WSNs. The network lifetime is finally stretched out by utilizing portability designs for base station. In [10], the drawbacks are there are no fixed energy levels for base station and it doesn't contain predefined process built-in network. The paper [11] utilizes the support vector regression technique to construct a raised advancement show, by which the ideal direction of the mobile sink can be resolved. The system lifetime is influenced by the direction. To amplify the system lifetime, the mobile sink in the event driven is utilized to gather the caught information of events. In [11], drawback of the system is decrease in network lifetime. In [12], the authors proposed a mobile data collection call for various sensor systems. Here [12], the drawback is usage of more number of mobile users in network for network process.

The paper [13] implemented for collecting mobile data consisting of the sensor layer, cluster head layer, and mobile collector layer in wireless sensor networks. The system employs LBC-DDU i.e., distributed load balanced clustering and dual data uploading. The reason for being developing such system is to achieve good scalability, long network lifetime and low data collection latency.

In [13], the authors proposed a heuristic tour planning algorithm to discover an obstruction maintaining a strategic distance from most limited route for the mobile sink. Keeping in mind the end goal is to better take care of the dispatch issue of the mobile sink, by utilizing this algorithm it possible to develop the spanning tree of the system model. As 
per the spanning diagram, all the obstructions are staying away from ways. Furthermore, the obstacle maintains smallest path for the mobile sink will be acquired from these obstacle avoiding paths. Paper presents particular steps of the heuristic obstacle avoiding calculation based algorithm below. Here [13], drawbacks are clustering head transmission time is more and mobile user takes more time to collect the data from cluster head.

In physical conditions, the detecting field may contain obstacles with various shapes and sizes. Due to the irregular shape of obstacles, it can't directly construct the deterrent spreading over diagram for the mobile sink scheduling on the basis of the spanning graph algorithm above. In this manner, an exploration challenge is how to utilize the spanning graph algorithm to discover an obstacle-avoiding shortest route for the mobile sink. The papers [14-16, 18] utilize network based systems to examine and tackle issues in WSNs. Here, by also utilizing grid based strategies to take care of the planning problem of the mobile sink. The detecting region is isolated into a similar size grid cells by using the grid-based techniques. Obviously, edges of obstructions meet grid cells and obstacles may have some part of grid cells. When obstacles involve some part of one grid cell, it is expected that the grid cell is viewed as obstacles. The drawback of [14-16, 18] is it doesn't avoid the obstacles in network with proper region. In [17], presented a procedure for building an energy effectiveness list tree, called EGF-tree, for sorting out sensor nodes into a tree progression, where sensor nodes are in skewness appropriation. In view of this EGF-tree pecking order, multidistrict inquiries are collected in-system and questions are executed proficiently. Here [17] has setup the sensor node into tree construction but it has a drawback in network because once construct the tree it can take multi-level communication.

In [19], OQoS-MRP is defined as link-based and path-based metrices. Each sensor is able to record the link performance between itself and its neighbor in terms of reliability, energy, delay, distance to sink and hop count. The path-based metrices are represented as $R_{\text {path }}, E_{\text {path }}$ and $D_{\text {path }}$. In [20], PSO (Particle Swarm Optimization) is employed for efficient clustering of nodes with least redundant node in the network. PSO is a computational technique that optimizes a problem using a series of iterations to enhance the possible solution for a given quality measure.

The proposed method utilizes the all users in network and plans to reduce the time interval based on criteria. Our method utilizes individual energy levels for each node and stores it after completion of cluster head rotation. Here, one mobile user is taken and is named as mobile sink so transaction period also can be decreased. After comparing with all existing convolution schemes, proposed method is better than all such criteria's and is described in detail in section 3 .

\section{Proposed framework}

In this segment, described proposed system CODS-KM, Collection Oriented Distributed Scheme for WSN with Distance, Energy, Link quality, Bandwidth, and Delay. Paper introduced clustering mechanism in previous work so paper should follow k-medoids algorithm for clustering and cluster head selection that works with CODS. Here all the communications are directed through cluster head $(\mathrm{CH})$ to $\mathrm{RP}$ (rendezvous point) called as collection point (CP) then directly goes to sink (receiving node). Here sink will act as Mobile sink to collect the information from collection points within short period. The proposed system presents, mitigation of travelling time during transmission and finally to collect all the information from collection points and continues up to end of simulation process.

During the clustering process, the nodes will compare their information with each other and designate the cluster heads. Once the cluster head is selected, then the cluster head node intimates it selection to all the other nodes and remaining nodes join as member. The difference between clustering algorithms are distance and energy. The k-medoids calculates distance between the sensor node and sink node and selects cluster heads.

To address the described issue, proposed approach works by partitioning the System, in each segment and mobile element is assigned. This apportioning contemplates the conveyance of the nodes, to maintain a strategic distance from long separation. Proposed approach begins; by distinguishing the arrangement of nodes, they are used to build mobile elements visits. To acquire these sets, utilization of the collection point based algorithm is proposed. Once the nodes of the visits are recognized, the proposed approach begins by apportioning the system into two segments. 


\section{Algorithm: Cluster Head Selection-CHS}

Step1: select initial medoids

1.1 Using Euclidean distance as a dissimilarity measure computes the distance between every pair of all objects as follows:

$\left\{d_{i j}=\sqrt{\sum_{a=1}^{p}\left(X_{i a}-X_{j a}\right)^{2}} \mathrm{i}=1, \ldots \ldots, \mathrm{n} ; \mathrm{j}=\right.$

$1, \ldots, \mathrm{n}\}$

$X_{i a} \quad------\rightarrow$ first point (x-topology)

representation

$X_{j a} \quad-----\rightarrow$ second point (y-topology)

represenation

a-- $\rightarrow$ initial value

$p-->>$ ending value

$d_{i j}-\rightarrow$ euclidean distance

1.2 Calculate $P_{i j}$ to make an initial guess at the centers of the clusters.

$$
\left\{P_{i j}=\frac{d_{i j}}{\sum_{l=1}^{n} d_{i l}} \mathrm{i}=1, \ldots . ., \mathrm{n} ; \mathrm{j}=1, \ldots ., \mathrm{n}\right\}
$$

$P_{i j}--\rightarrow$ medoid point

1.3 At each object, calculate $\sum_{i=1}^{n} d_{i j}(\mathrm{j}=$ $1, \ldots, n)$ and arrange them in ascending order. Select k objects as initial group medoids which are having the minimum value.

1.4 Set to the nearest medoid for each object.

1.5 Determine the sum of distance from all objects to their medoids i.e., the current optimal value.

Step2: Find out new medoids

The current medoid which minimizes the total distance to other objects in its cluster is replaced by the object in each cluster.

2.1 To the nearest new medoid, each object is assigned.

2.2 Determine the sum of distance from all objects to their new medoids which is called the new optimal value. If the optimal value is equal to the previous one then stop the algorithm else, go back to the Step 2.

\section{Algorithm: Cluster-based Rendezvous collection points \\ Input:}

Set of collection points CPS $\left(C P_{0}, \ldots, C P_{\mathrm{n}-1}\right)$

CPS ---> collection point set

$C P_{0}$---->initial collection point

$C P_{\mathrm{n}-1}---->>$ ending collection point

Transmission range----> $T_{r}$

Output

Set of optimized collection points

Start:

1. for $i=0$ to $n-2$

2. begin

3. if dist $\left(C P_{i}, C P_{i+1}\right) \leq T_{r}$

4. $C L P \leftarrow C L P-C P_{i+1} \quad ; C L P-$ $>$ particular collection point

5. End if

6. Else if collection points $c_{i+1}$ to $c_{j}$ have common intersection area

7. else if dist $\left(c_{i+1}, c_{i+2}\right) \leq T_{r}$

8. begin

9. $k \leftarrow i+1$

10. while collection points from $c_{i+1}$ to $c_{k+1}$ have common intersection area

11. begin

12. $k \leftarrow k+1$

13. End while

14. a point $r_{i+1}$ within the common intersection area nearer to the path from $c_{i}$ to $c_{k}$ will be the rendezvous point

15. $C L P \leftarrow C L P-\left\{c_{i+1} . . c_{k-1}\right\}$

16. $C L P \leftarrow C L P \cup r_{i+1}$

17. end if

18. else if per_dist $\left(c_{i}, c_{i+1}, c_{i+2}\right) \leq T_{r}$

19. begin

20. $k \leftarrow i+1$

21. while the path from $c_{i}$ to $c_{k+2}$ covers set of collection points $\left\{c_{i+1} \ldots c_{k+1}\right\}$

22. begin

23. $k \leftarrow k+1$

24. end while

25. $C L P \leftarrow C L P-\left\{c_{\mathrm{i}+1} \ldots c_{k-1}\right\}$

26. end if

27. else

28. begin

29. $r_{i+1} \leftarrow$ point_at_dist $\left(c_{i+1}, \mathrm{c}_{i+2}, T_{r}\right)$

30. $C L P \leftarrow C L P-c_{i+1} \cup r_{i+1}$

31. end else

32. end for

33. RPS $\leftarrow$ Lin - Kernighan $(C L P) / /$

Shortest path using Lin-Kernighan End

Continues.... 


\begin{tabular}{|c|c|}
\hline \multicolumn{2}{|l|}{ Algorithm:dist $\left(P_{1}, P_{2}\right)$} \\
\hline Input & \\
\hline 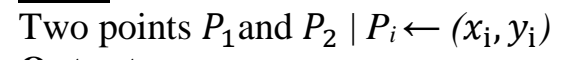 & \\
\hline Output & \\
\hline Distance d between points $P_{1}$ and $P_{2}$ & \\
\hline Start: & \\
\hline$d=\sqrt{\left(x_{2}-x_{1}\right)^{2}+\left(y_{2}-y_{1}\right)^{2}}$ & \\
\hline Return $(d)$ & \\
\hline End & \\
\hline
\end{tabular}

The dividing step utilizes the outstanding kmedoid calculation. Once these two apportioned are acquired, the procedure continues by building a solitary visit for each partition. The tours inside each partition are constructed using Christofides algorithm. For each segment, if they got call that fulfills the time-due date requirement, this tour will be assign to a mobile element. Something else, this partition will be re-divided and the visit development step will be retriggered.

$\mathrm{K}$-medoids clustering is a variation of $\mathrm{K}$-means that is more powerful to noise and exceptions. Rather than utilizing the mean point as the central point of a cluster, K-medoids utilizes an original point in the group of cluster to indicate it. Medoids is the most intermediate discoverable phenomenon of the cluster, with minimum aggregate of separations to various points.

\section{Experimental results}

In this paper, assumption of 25 sensor nodes and they are randomly distributed over a $1000 \times 500 \mathrm{~m}^{2}$ area where different random way points setup. By considering this research, there is no gap exists in the detected space as well as static sensors are same in abilities. In the meantime, the mobile sink is located in the top-left corner of the two-dimensional territory and its coordinates are $(50 \mathrm{~m}, 50 \mathrm{~m})$. Here, consideration of static network scenario with nodes randomly distributed. The mobile sink starts its periodical avoiding path from its initial site and finally returns. In proposed framework, network size is more, data storage space high, collection points with high efficiency and less energy consumption compared to previous methods. Proposed collection oriented routing approach CODS-KM is compared with Heuristic tour planning algorithm [13] and LEACH [18] without network failure using 25 nodes in the network. The proposed method consists of clusters, cluster heads and collection points. Here proposed system time duration for individual nodes and setup the configuration process of individual clusters. The data delivery process through cluster heads sent to mobile sink. The selection of cluster head is based on k-medoids algorithm. In this proposed framework distance, energy, link factor and delay parameters are applied to our network users then executed. The distance parameter is helpful for cluster head selection. The individual node energy level knows the strength of node. The link factor between two users is calculated and delay finds the how much time is taken for transmission process. Proposed method allocates mobility time for mobile sink and increases the lifetime of network. The performance evaluation metrics used in the simulation are End to End Delay, Energy consumption, Travelling time and Throughput. The system parameters used in simulations are mentioned in Table 1. In this paper, to improve planning for the mobile sink, acknowledged that the data accumulated by sensor nodes is the deferral progressive data, i.e., they can hold for the mobile sink to get and bring them up.

Evaluation results: In this section, the evaluation metrics used in the evaluation of proposed approach is given: SACO-KM-ECC.

\section{End2End Delay is defined as}

Delay (i) $=$ receiving time (i) - sending time (i)

Total delay $=$ Total delay + delay $(\mathrm{i})$

$$
\text { Average delay }=\frac{\text { Total Delay }}{\text { count }}
$$

Here

Count $=$ Total packet count

$\mathrm{i}=$ Packet sequence number

\section{Energy consumption is defined as}

$$
\begin{aligned}
& \text { Energy Consumption (i) }= \\
& \quad \text { initial energy (i) - final energy (i) }
\end{aligned}
$$

\section{Throughput is defined as}

Thr

$=($ Received data $\times 8) /($ Data transmission period $)$

Table1. System parameters

\begin{tabular}{|l|l|}
\hline PARAMETER & VALUE \\
\hline Application Traffic & CBR \\
\hline Transmission rate & 15 packets $/ \mathrm{sec}$ \\
\hline Radio range & $250 \mathrm{~m}$ \\
\hline Packet size & 512 bytes \\
\hline Maximum speed & $30 \mathrm{~m} / \mathrm{s}$ \\
\hline Simulation time & $8000 \mathrm{~ms}$ \\
\hline Number of nodes & 25 \\
\hline Area & $1000 \times 500$ \\
\hline Grid size & $10 \mathrm{~m}$ \\
\hline
\end{tabular}




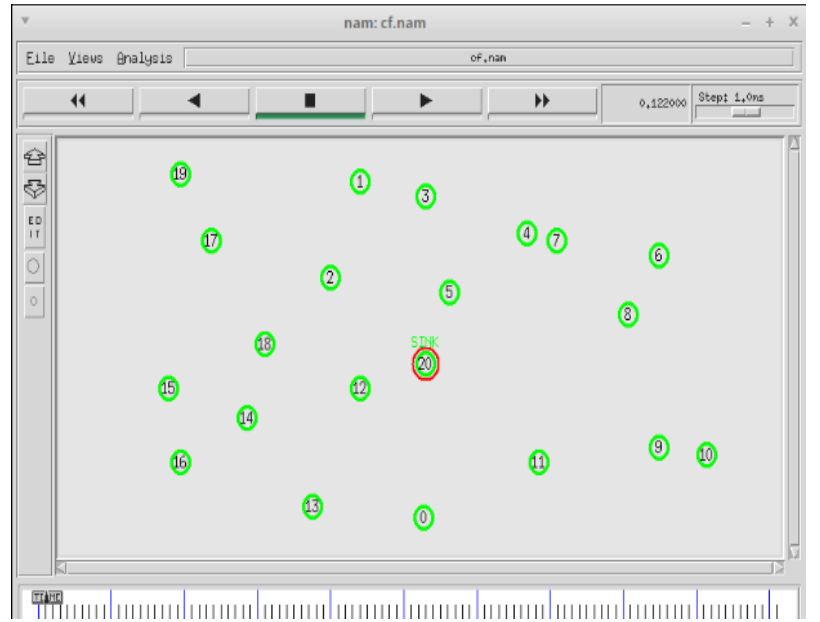

Figure. 1 Network deployment

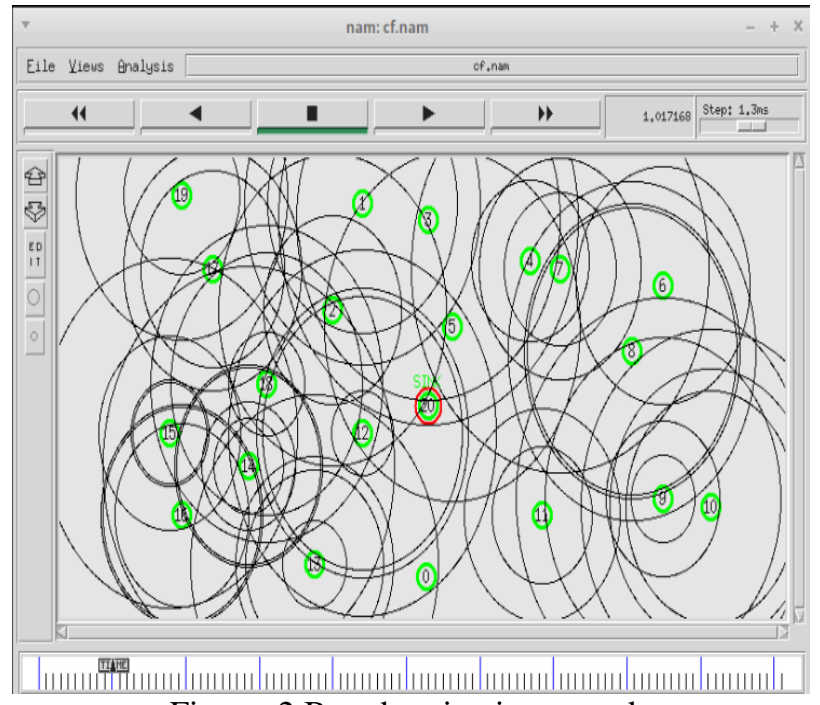

Figure. 2 Broadcasting in network

In this area, used heuristic obstacle avoidance based algorithm to lead various analyses in detecting field with obstacles. As indicated by the system lifetime and the development way of mobile sink, exploratory outputs of the calculation which are presented below.

Fig. 1 represents all nodes arranged in system and organization of nodes in a network accordingly. Here all nodes are displayed depending on topology results and all properties of NAM window. In this network, considered area is $1000 \times 500$. Here different attributes, variables are used and they show how to perform network. Considered 25 nodes and given topology values for each node and they are for communication purpose. In the simulation network, parameters considered are simulation time, step time and distance.

Fig. 2 shows the occurrence of broadcasting throughout the network and it is for communication purpose. As per the simulation result in Fig. 2, all

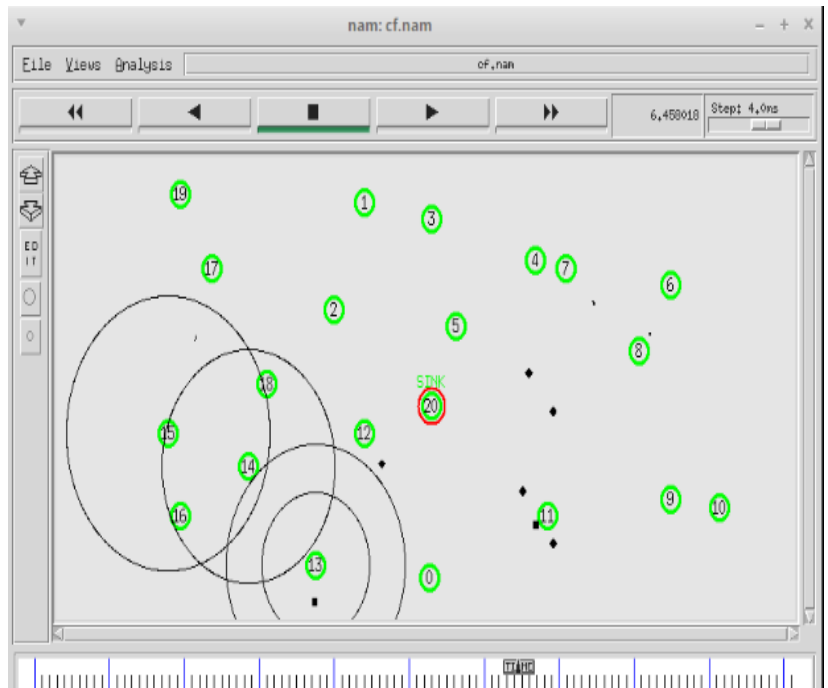

Figure. 3 Packet loss due to heavy traffic

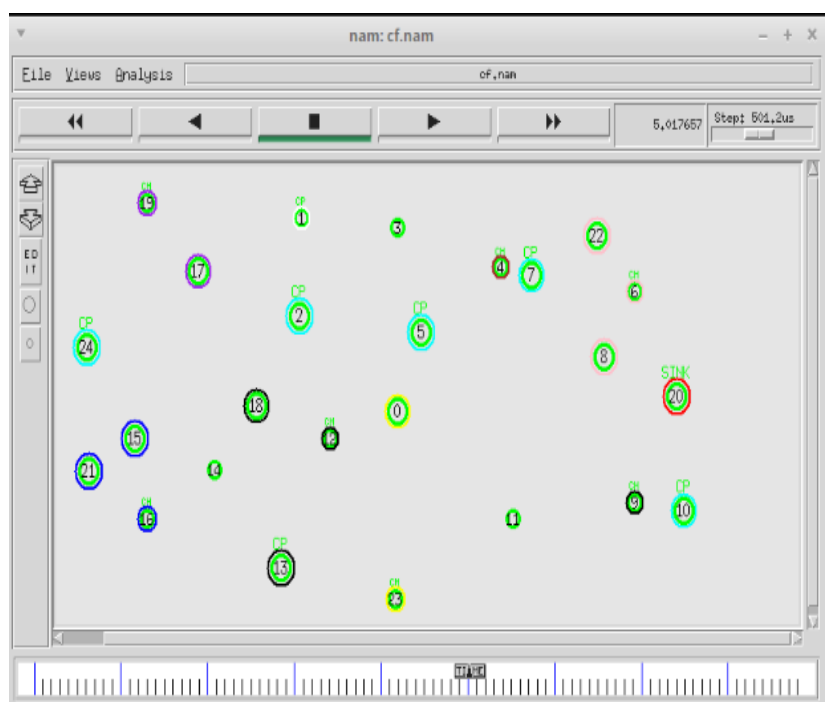

Figure. 4 Proposed network deployed

nodes are involved in this process. Broadcasting process starts from source node and it is communicating with remaining nodes. The network process is depends on broadcasting. The source node starts the process of broadcasting and it is continued in all the levels in network.

Fig. 3 shows the packet loss due to the traffic during communication process. In simulation process, packet loss can be reduced using cluster based approaches. So it is used to discriminate the traffic.

In this paper, introduced new mechanism and it named as CODS-KM. Here all nodes are represented based on topology values. In proposed network, 1000x500 area considered and all the nodes are arranged as different clusters. So here total number of clusters is eight. In these clusters, one of the nodes is chosen as cluster head and this 


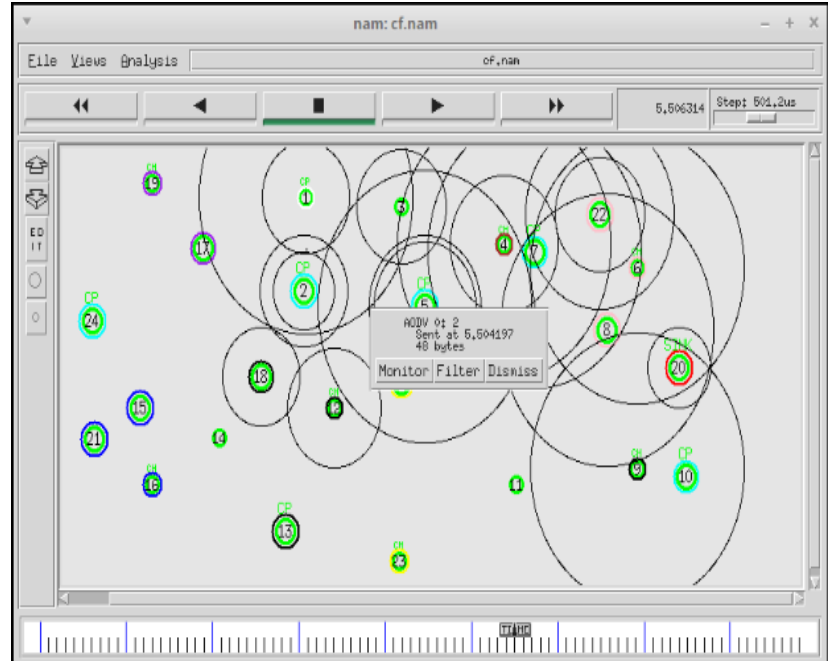

Figure. 5 Broadcasting in proposed system

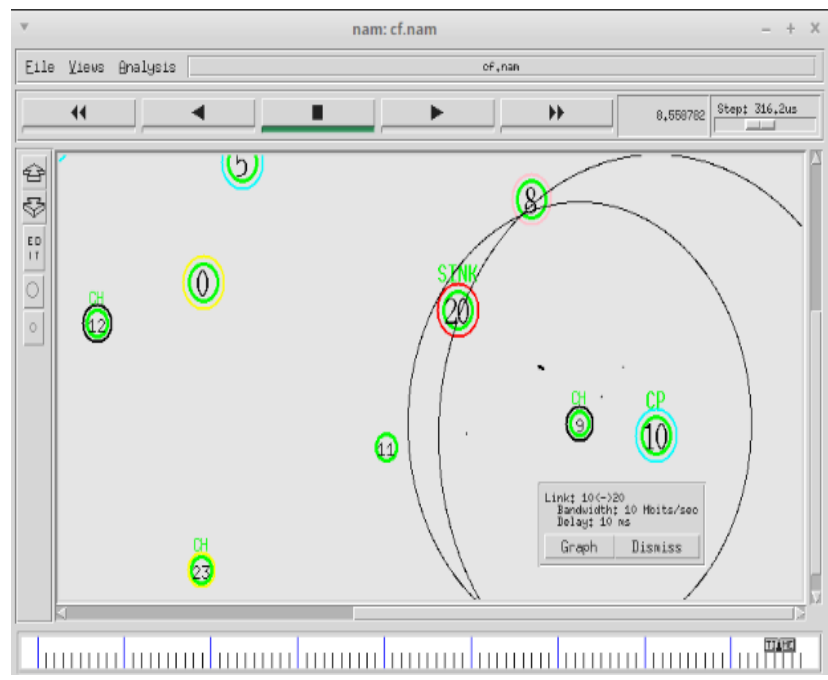

Figure. $6 \mathrm{CP}-10$ to Mobile sink data process

cluster head communicates with remaining cluster nodes. Our proposed network deployment is shown in Fig. 4.

Fig. 5 indicates broadcasting of data for next communications. It is observed clearly, there is a proper communications for data sharing and much number of packets is in delivering process. Proposed mechanism (CODS-KM) is providing optimal paths and securing communication levels. Fig. 5 shows the proposed mechanism named as a collection point scheme add into heuristic algorithm.

In this network, the data sharing process through different nodes is displayed and the routing process occurs based on clusters. Every cluster head communicates with cluster nodes. Whenever clustering process is completed immediately proposed mechanism process starts and communication between cluster head and collection point starts the process of data sharing. Proposed mechanism supports the routing process. Here network performance improved and all the nodes are properly communicating for best performance in routing and it is represented in Fig. 6.

Fig. 7 represents trace file of the proposed system from initialization to end of network process. All the nodes, variables, values, parameters, routing levels, and time variation depends on the network process and all trace from a proposed framework network animator (NAM). Here trace file indicates an output file of network.

In above Fig. 8 end2end delay is presented and the graph is plotted between simulation time versus delay. CODS-KM method improves delay time it means decrease in the delay between communications nodes and it is better when compared to heuristic-tour planning algorithm, normal cluster-method (LEACH) and OQoS-MRP. In proposed network, one can easily how much time is taken for completion of routing process and delay time also.

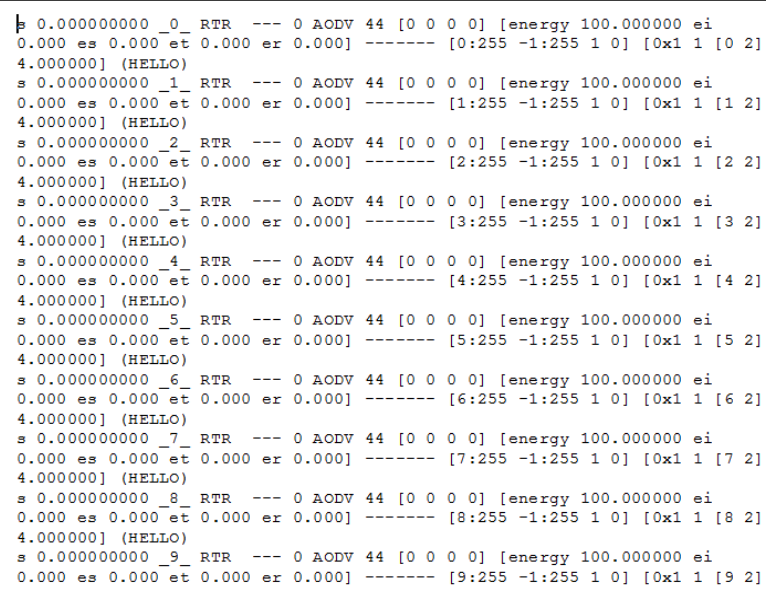

Figure. 7 Trace file of proposed framework

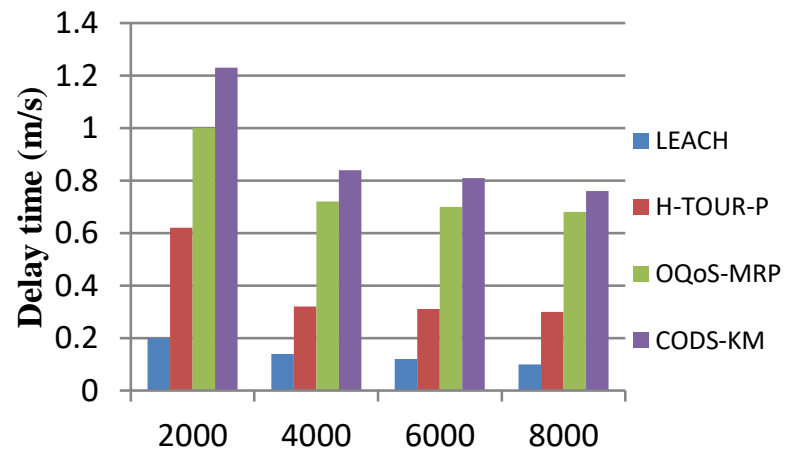

Time (msec)

Figure. 8 Performance on delay 


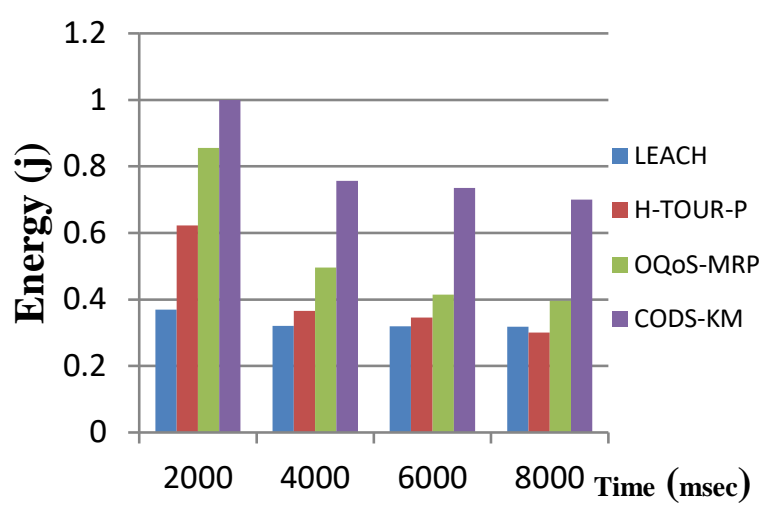

Figure. 9 Energy level routing

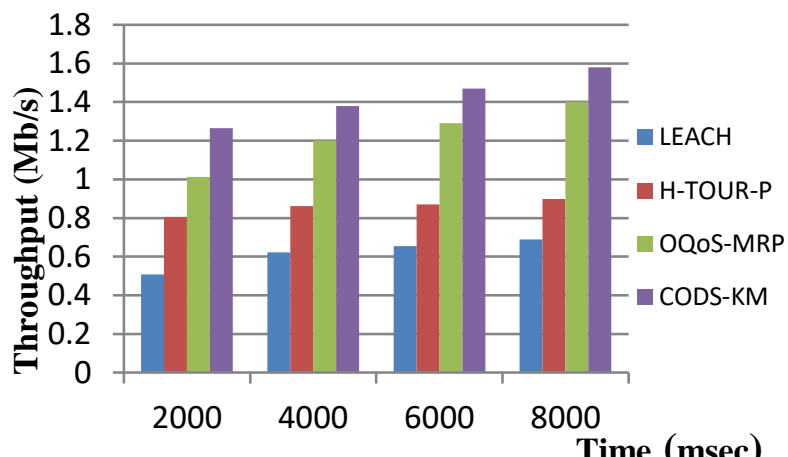

Figure. 10 Network performance

Fig. 9 represents the graph between simulation time versus energy. It is found that CODS-KM method improves the energy values, when compared to heuristic-tour planning algorithm, normal clustermethod (LEACH) and OQoS-MRP. Fig. 9 represents the usage of data energy levels proposed network. In this plot, communication levels indicate energy levels of data and verifies the node capability based on these energy levels. From network simulation, decrease in energy consumption is leads to increase of network lifetime.

The plot between simulation time versus throughput is shown in Fig. 10. It is found that CODS-KM method improves the throughput when compared to heuristic-tour planning algorithm, normal cluster-method (LEACH) and OQoS-MRP. In Fig. 10, the comparison done between proposed and existing frameworks (heuristic-tour planning algorithm and normal cluster-method (LEACH)). The performance of network is easily observed by studying network throughput which is shown in Fig. 10 .

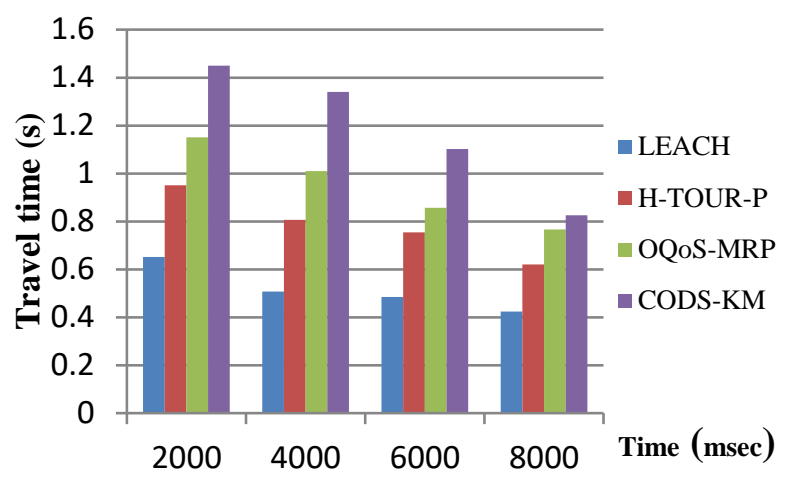

Figure. 11 Travelling time in routing

The above Fig. 11 shows the graph between simulation time versus travelling time. CODS-KM method decreases the travelling time when compared to heuristic-tour planning algorithm, normal cluster-method (LEACH) and and OQoSMRP. It is clearly observed in Fig. 11. The travelling time for routing process decreased and it benefits to improve the network efficiency.

\section{Conclusion}

In this paper, proposed a model CODS-KM, Collection oriented distributed scheme with kmedoids clustering approach and considered parameters like Distance, Energy, Link quality and Delay. In CODS-KM, routing decisions are based on Collection point methodology which incorporates Remaining Energy, Distance, and Link quality, which attributes to choose the best next hop for the routing operation, thus allowing for better load balancing and network lifetime extension. Here KMedoids function is useful for selection of clusterhead. In this paper, mobile sink is used to extend the lifetime of a network. Based on the cluster-based method, used the heuristic obstacle-avoiding calculation based algorithm to release the mobile sink. The paper proposes a collection oriented distributed scheme to limit the quantity of got visits. For simulation verification, NS2 software is used and exploratory outcomes demonstrate that our cluster-based approach is practical for sending mobile sink. The simulation results and different graphical analysis like delay time, energy consumption, throughput and travelling time are presented in section 4 . The major contribution of this research is to minimize the travelling time in WSN. So, the proposed framework is best for maintaining time interval and minimizing it. The future extent of this paper is bunch heads whatever 
gathered information specifically advances to versatile sink and also accumulation focuses.

\section{References}

[1] J. C. Cuevas-Martinez, J. Canada-Bago, J. A. Fernandez-Prieto, and M. A. Gadeo-Martos, "Knowledge-based duty cycle estimation in wireless sensor networks: Application for sound pressure monitoring", Applied Soft Computing, Vol. 13, No. 2, pp. 967-980, 2013.

[2] H.-L. Fu, H.-C. Chen, and P. Lin, "Aps: Distributed air pollution sensing system on wireless sensor and robot networks", Computing Communications., Vol. 35, No. 9, pp. 1141-1150, 2012.

[3] Z. Shen, X. Pan, C. Huang, J. Feng, Y. Zhao, M. Gao, and L. M. Ni, "Energy consumption monitoring for sensor nodes in SNAP", International Journal of Sensor Networks, Vol. 13, No. 2, pp. 112-120, 2013.

[4] B. Zhou, S. Yang, T. H. Nguyen, T. Sun, and K. T. V. Grattan, "Wireless Sensor Network Platform for Intrinsic Optical Fiber pH Sensors", IEEE Sensors Journal, Vol. 14, No. 4, pp. 1313-1320, 2014.

[5] M. Dong, X. Liu, Z. Qian, A. Liu, and T. Wang, "QoE-ensured price competition model for emerging mobile networks", IEEE Wireless Communications, Vol. 22, No. 4, pp. 50-57, 2015.

[6] S. Gowrishankar, T.G. Basavaraju, D.H. Manjaiah, and S.K. Sarkar, "Issues in Wireless Sensor Networks", In: Proc. of the World Congress on Engineering, Vol I, 2008.

[7] W. B. Heinzelman, A. P. Chandrakasan, and H. Balakrishnan, "An application-specific protocol architecture for wireless microsensor networks", IEEE Transactions on Wireless Communications, Vol. 1, No. 4, pp. 660-670, 2002.

[8] G. Smaragdakis, I. Matta, and A. Bestavros, "SEP: A stable election protocol for clustered heterogeneous wireless sensor networks", In: Proc. of SANPA, pp. 1-11, 2004.

[9] A. W. Khan, A. H. Abdullah, M. A. Razzaque, and J. I. Bangash, "VGDRA: A virtual grid-based dynamic routes adjustment scheme for mobile sink-based wireless sensor networks", IEEE Sensors Journal, Vol. 15, No. 1, pp. 526-534, 2015.

[10] O. Cayirpunar, E. Kadioglu-Urtis, and B. Tavli, "Optimal base station mobility patterns for wireless sensor network lifetime maximization", IEEE Sensors Journal, Vol. 15, No. 11, pp. 6592-6603, 2015.

[11]F. Tashtarian, M. H. Y. Moghaddam, K. Sohraby, and S. Effati, "On Maximizing the Lifetime of Wireless Sensor Networks in Event-Driven Applications With Mobile Sinks", IEEE Transactions on Vehicular Technology, Vol. 64, No. 7, pp. 3177-3189, 2015.

[12] M. Ma, Y. Yang, and M. Zhao, "Tour planning for mobile data-gathering mechanisms in wireless sensor networks", IEEE Transactions on Vehicular Technology, Vol. 62, No. 4, pp. 1472-1482, 2013.
[13] G. Xie and F. Pan, "Cluster-Based Routing for the Mobile Sink in Wireless Sensor Networks With Obstacles", IEEE Access, Vol. 4, pp. 2019-2028, 2016.

[14] C. Ai, L. Guo, Z. Cai, and Y. Li, "Processing Area Queries in Wireless Sensor Networks", In: Proc. of the Fifth International Conference on Mobile Ad-hoc and Sensor Networks, pp. 1-8, 2009.

[15]I.-H. Peng and Y.-W. Chen, "Energy consumption bounds analysis and its applications for grid based wireless sensor networks", Journal of Network and Computer Applications, Vol. 36, No. 1, pp. 444-451, 2013.

[16] K. Xu, G. Takahara, and H. Hassanein, "On the robustness of grid-based deployment in wireless sensor networks", In: Proc. of the Int. Conference Wireless Communications and Mobile Computing, pp. 1183-1188, 2006.

[17]Z. Zhou, J. Tang, L.-J. Zhang, K. Ning, and Q. Wang, "EGF-tree: An energy-efficient index tree for facilitating multi-region query aggregation in the Internet of Things", Personal and Ubiquitous Computing, Vol. 18, No. 4, pp. 951-966, 2014.

[18] G. Han, A. Qian, J. Jiang, N. Sun, and L. Liu, “A grid-based joint routing and charging algorithm for industrial wireless rechargeable sensor networks", Computer Networks, Vol. 101, pp. 19-28, 2016.

[19]D. Onthachi, and S. jayabal, "An Optimized Qosbased Multipath Routing Protocol for Wireless Sensor Networks", International Journal of Intelligent Engineering and Systems, Vol.11, No.2, pp. 49-56, 2018.

[20] N. Hiremani and T. G. Basavaraju, “An Efficient Routing Protocol Adopting Enhanced Cluster Formation Technique Accompanied by Fuzzy Logic for Maximizing Lifetime of WSN", International Journal of Intelligent Engineering and Systems, Vol.9, No.4, pp.185-194, 2016. 